\title{
Challenges of Generating and Maintaining Protective Vaccine-Induced Immune Responses for Foot-and-Mouth Disease Virus in Pigs
}

\author{
Nicholas A. Lyons ${ }^{1,2 *}$, Young S. Lyoo ${ }^{3}$, Donald P. King ${ }^{1}$ and David J. Paton ${ }^{1}$ \\ ${ }^{1}$ The Pirbright Institute, Pirbright, UK, ${ }^{2}$ European Commission for the Control of Foot-and-Mouth Disease, Food and \\ Agriculture Organization of the United Nations, Rome, Italy, ${ }^{3}$ College of Veterinary Medicine, Konkuk University, Seoul, \\ South Korea
}

OPEN ACCESS

Edited by:

Andres M. Perez,

University of Minnesota, USA

Reviewed by:

Danilo Bucafusco,

National Agricultural Technology

Institute, Argentina

Carolina Stenfeldt,

Agricultural Research Service, United States Department of

Agriculture, USA

*Correspondence:

Nicholas A. Lyons

nick.lyons@pirbright.ac.uk

Specialty section:

This article was submitted to

Veterinary Epidemiology and

Economics,

a section of the journal

Frontiers in Veterinary Science

Received: 02 September 2016 Accepted: 01 November 2016 Published: 30 November 2016

Citation:

Lyons NA, Lyoo YS, King DP and

Paton DJ (2016) Challenges of

Generating and Maintaining

Protective Vaccine-Induced Immune

Responses for Foot-and-Mouth

Disease Virus in Pigs.

Front. Vet. Sci. 3:102.

doi: 10.3389/fvets.2016.00102
Vaccination can play a central role in the control of outbreaks of foot-and-mouth disease (FMD) by reducing both the impact of clinical disease and the extent of virus transmission between susceptible animals. Recent incursions of exotic FMD virus lineages into several East Asian countries have highlighted the difficulties of generating and maintaining an adequate immune response in vaccinated pigs. Factors that impact vaccine performance include (i) the potency, antigenic payload, and formulation of a vaccine; (ii) the antigenic match between the vaccine and the heterologous circulating field strain; and (iii) the regime (timing, frequency, and herd-level coverage) used to administer the vaccine. This review collates data from studies that have evaluated the performance of foot-and-mouth disease virus vaccines at the individual and population level in pigs and identifies research priorities that could provide new insights to improve vaccination in the future.

Keywords: foot-and-mouth disease, pigs, vaccination, immunity

\section{INTRODUCTION}

Foot-and-mouth disease (FMD) is a viral disease of cloven-hooved animals causing severe economic impacts (1). The disease circulates widely in sub-Saharan Africa and Asia, but has been largely eradicated from South America as well as much of the developed world. It is caused by a Picornavirus (FMD virus: FMDV) that exists as seven immunologically distinct serotypes. Global FMD control efforts are focused at reducing the burden of disease, with the longer-term goal to sequentially eliminate the virus from livestock populations. Vaccination can be a highly effective tool to control FMD, especially when it is implemented together with effective zoo-sanitary measures (farm biosecurity and quarantine) and culling of infected animals. During the 1980s, vaccines were used to effectively eradicate FMD from continental Europe (2), and, more recently, FMD control in South America has employed extensive use of vaccination (3).

In attempts to maximize the impact of limited vaccine resources, most FMD control programs emphasize the use of FMDV vaccines in cattle. As a consequence, many of the published studies that evaluate FMDV vaccine performance have also focused exclusively on their use in cattle. However, some countries have large pig populations that are a major target for FMDV vaccination. The impact of FMD in pigs has recently become particularly important in many Asian countries, such as China 
and the Republic of Korea, where there have been extensive and sustained FMD outbreaks due to serotype $\mathrm{O}$ and $\mathrm{A}$ lineages that have emerged from mainland Southeast Asia $(4,5)$. The continued occurrence of FMD cases in countries that have large pig populations despite extensive vaccination has raised questions about the effectiveness of vaccination in pigs, but published field studies that analyze this issue appear to be lacking. This review highlights the difficulties of FMDV vaccination in pigs at the individual and population level and summarizes the studies that have evaluated the performance of FMDV vaccines in this important domesticated livestock species.

\section{GENERAL CONSIDERATIONS FOR FMD VACCINATION}

\section{Types of Vaccines in Commercial Use Today in Pigs}

Foot-and-mouth disease vaccines have been produced on a large scale since the 1940s (6) and are currently manufactured by at least 56 commercial and governmental institutions around the world (O Mezzer, Vallée SA, Personal Communication, 2014). In all FMDV susceptible species, there are many types of vaccine available, not just by virtue of the serotypes and strains included, but also the adjuvant (aluminum hydroxide/saponin or oil adjuvants as: oil in water, water in oil, and double water emulsion) and inactivation method (binary ethyleneimine or rarely formaldehyde) (7). For pigs, currently available vaccines are formulated with an oil adjuvant, due to poor immunogenicity with the aqueous equivalents, and contain either killed/inactivated FMD virus or a synthetic viral peptide $(8,9)$.

\section{Reasons for Vaccine Failure}

There are a number of problems with current FMD vaccines that limit their effective use. These include: imperfect antigenic match between the field virus and vaccine strain; variable antigenic payload; antigen instability (principally the $146 \mathrm{~S}$ virus particles); requirement for a cold-chain; poor adaptation of certain strains for vaccine production; short duration of protection and requirements for repeat boosting; non-sterile immunity with clinically protected animals sometimes becoming infected; high levels of coverage required for herd immunity; and interference by maternally derived antibody $(10,11)$. Despite these problems, FMD vaccines can play a vital role in disease control and are very widely used, with over two billion doses estimated to be used globally each year (12). The general reasons for vaccination failure have been helpfully summarized by Heininger et al. (13). "Vaccine failure" may be related to the recipient (pig) or the actual vaccine. "Failure to vaccinate" can be due to errors in vaccine use by the user and program-related problems. In the context of porcine FMD vaccines, these key issues are summarized in Figure 1.

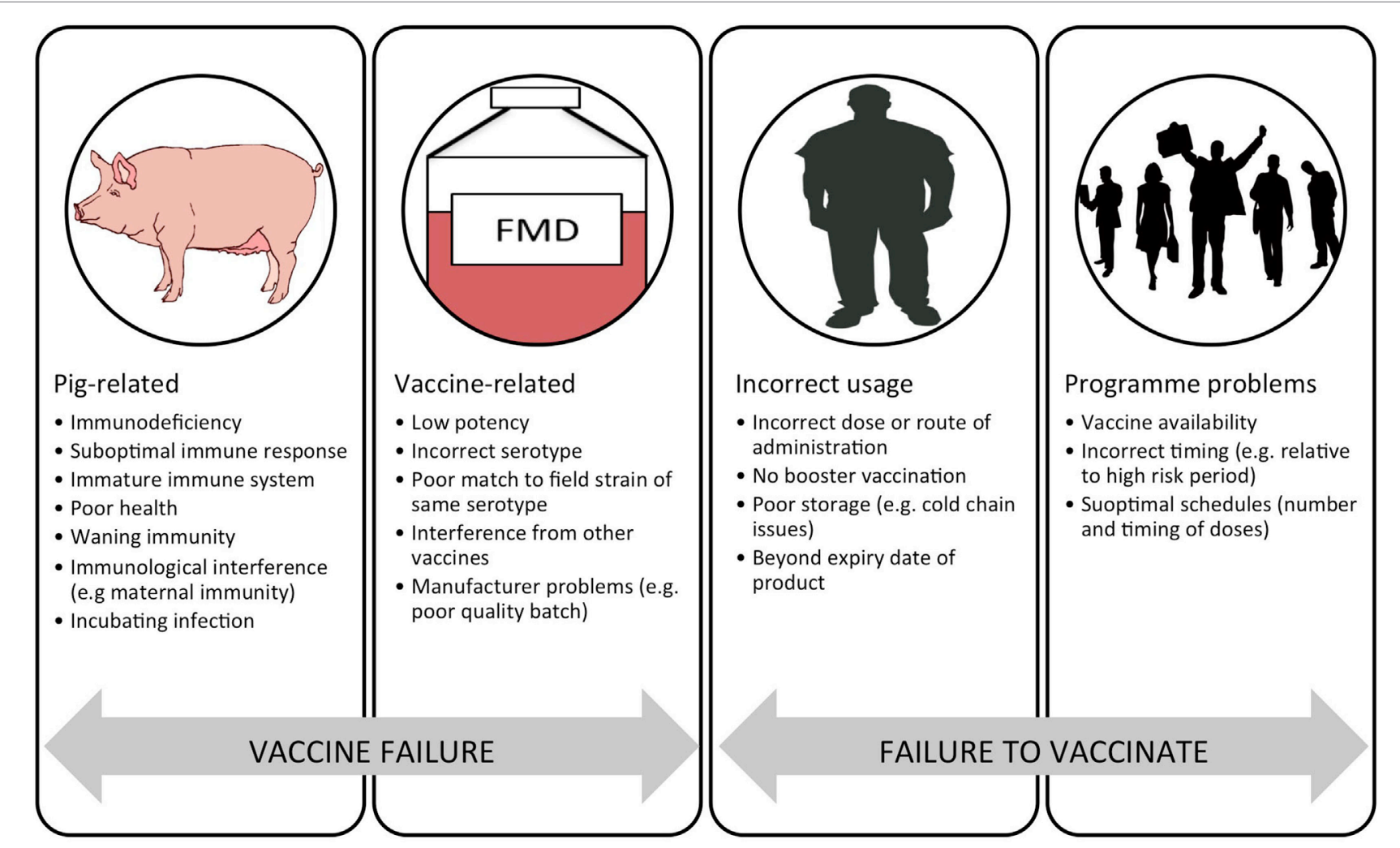

FIGURE 1 | Schematic representation of the reasons for a failure in vaccination divided into "vaccine failure" and "failure to vaccinate." Adapted from Ref. (13). 


\section{IMMUNITY AND IMMUNOGENICITY}

Comparative interpretation of reports on the evaluation of FMD vaccines are often complicated by significant differences in the potency and other characteristics (e.g., different adjuvants and oil emulsions) of the different vaccines under study, as well as different methods and severity of challenge models (mainly direct or indirect contact with infected unvaccinated or vaccinated donors or intramuscular or intradermal inoculation). The immune responses of pigs to FMD vaccines are less well studied than those of cattle (e.g., details of antibody isotypes, of local immunity, of breadth of antigenic protection, and of the correlation between antibody responses and protection), and there are few field study reports on vaccinated pigs (14). As for cattle and other species, establishing reliable correlates of serological protection for easy interpretation of field studies on vaccine-induced immunity in pigs are hampered by their dependence on specific attributes of the tests, vaccines, and challenge viruses involved.

\section{Immune Response to FMD Vaccines in Pigs}

Inactivated oil-adjuvanted FMD vaccines elicit antibody responses in pigs, and the extent of seroconversion measured by virus neutralization and liquid phase blocking ELISA (LPBE) tests can help to predict clinical protection (15-17). Eblé et al. (18) showed that reduced virus shedding was also correlated to neutralizing antibody levels induced by vaccination and that vaccine-induced mucosal IgA was associated with reduced susceptibility to infection. Cox et al. (19) showed that pigs immunized with highpotency vaccines could be protected against challenge 7 months later, associated with sustained levels of neutralizing antibody and a sustained increase in some cytokine levels in serum (IL-6, IL-8, and in some pigs IL-12). Compared to unvaccinated pigs, vaccinated animals that became infected had lower and shorter lived antibody responses to FMDV non-structural proteins $(18,20)$.

High potency vaccines can protect pigs by $\sim 4$ days after vaccination, before the development of appreciable antibodies (21) and, as for cattle, there appears to be a gray zone where the protection afforded by low levels of antibody is unpredictable (17). This suggests that other factors are involved in protective immunity. Systemic levels of some cytokines have been shown to increase following FMD vaccination in pigs $(22,23)$, and Rigden et al. (24) showed enhanced chemotaxis of cells of the innate immune defenses. Furthermore, the induction of both cellular and humoral arms of the immune system postvaccination has been demonstrated by measuring Th1 [interferon (IFN) gamma] and Th2 (IL-10) responses (25). Zhang et al. (17) studied cell-mediated immunity in 30 vaccinated and 3 unvaccinated pigs given three different doses of vaccine and challenged intramuscularly with 1000 pig ID50 at 28 days post vaccination (dpv). Twenty-five pigs had antibody levels measured by LPBE that could be associated with protection or not (the gray zone). Protection was associated with vaccine-induced increases in cytotoxic $\mathrm{T}$ cell numbers and in levels of IFN gamma, IL-12, and IL-15 in serum. Garcia-Briones et al. (26) reported that a recombinant vaccinia virus expressing the FMDV 3D protein could partially protect pigs through a cell-mediated mechanism in the absence of a humoral antibody response to FMDV.

\section{VACCINE POTENCY AND PROTECTION}

Potency is defined by the OIE as the "concentration of the immunologically active component" (27). Potency according to this definition is often measured by vaccine manufacturers through the quantification of antigen so that a dose of a vaccine delivers a known antigen "payload." The conventional method of evaluating the effectiveness of FMD vaccines is by experimentally challenging vaccinated and unvaccinated control animals. Although inconsistent with the OIE definition of potency, these evaluations are commonly known as "potency tests." The first of these tests estimates the $50 \%$ protective dose $\left(\mathrm{PD}_{50}\right)$ value and is also the recommended European Pharmacopeia (EP) test. The $\mathrm{PD}_{50}$ value is defined as the dose that protects $50 \%$ of those under the particular challenge regimen (28). The second OIE-approved test is the "Protection against Podal Generalisation" (PPG) method, which is commonly used in South America.

In the 2009 OIE guidelines, there are descriptions of protocols for calculation of the $\mathrm{PD}_{50}$ and PPG based on challenge experiments in pigs which are very similar to those described in cattle. For the $\mathrm{PD}_{50}$, three groups of five pigs, no younger than 2 months of age and free of FMD serum antibody, are given either a full dose, quarter dose, or 1/16th dose. They are challenged 28 days later by intradermal inoculation of $10,000 \mathrm{TCID}_{50}$ of the vaccine strain into one of the heel bulbs of the foot. Two unvaccinated control pigs are included for comparison and to demonstrate a consistent phenotype of the challenge strain. For the $\mathrm{PD}_{50}$ test, the main difference with the pig protocol is the route of inoculation, as cattle are challenged via the intradermolingual route. A PPG equivalent, whereby 16 animals are challenged after receiving a full dose is also described. These descriptions were not included in the 2015 version of this document that states "In general, a successful test in cattle is considered to be sufficient evidence of the quality of a vaccine to endorse its use in other species. Under circumstances where a vaccine is produced for use primarily in a species other than cattle, it may be more appropriate to potency test the vaccine in that same species" (27). Li et al. (29) have proposed an easier approach to inoculation by challenging intramuscularly behind the ear although a suckling mice passaged strain was needed over a conventional cell passaged version. In China, intramuscular inoculation of 1000 pig ID50 of challenge virus is widely used, as described in studies to evaluate novel vaccines (see section below).

\section{Transmission Studies}

There are numerous examples of challenge studies in pigs in the scientific literature to either evaluate the clinical protection afforded by vaccines or their potential role in reducing transmission. Salt et al. (21) evaluated a high potency, oil-based, monovalent serotype $\mathrm{C}$ vaccine (strain Oberbayern) by exposing groups of three non-vaccinated or vaccinated pigs to infected animals at $4,8,12,16$, and $21 \mathrm{dpv}$. The challenge virus was homologous to the vaccine strain. Contact was indirect to simulate airborne transmission and looked at both "water-in-oil-in-water" and 
"oil-in-water" vaccines. All unvaccinated controls showed generalized disease, but all vaccinated animals were protected from clinical disease. Li et al. (29) reported the findings of a homologous PPG test for a serotype $O$ strain using 16 vaccinated pigs and 3 unvaccinated controls that were challenged intramuscularly behind the ear, $28 \mathrm{dpv}$. All vaccinated animals were protected from clinical disease, and the authors stated that two of the three controls had to show clinical disease for the test to be valid.

Eblé et al. (30) used challenge studies to estimate the impact of vaccination on transmission within pens in a high containment unit using a serotype $\mathrm{O}$ Taiwan strain. The vaccine was a double oil emulsion containing $3 \mu \mathrm{g}$ of $146 \mathrm{~S}$ antigen per dose. A single animal in a group of six was challenged by intradermal inoculation in the heel bulb, 7 or $14 \mathrm{dpv}$. Transmission to the in-contact pigs was evaluated by observing clinical signs, seroconversion to NSP antibodies, and detecting virus in oral swabs and serum. Three of the five contact animals in the 7-day group showed generalized clinical disease compared to none of those in the 14-day group. Additionally, no virus could be detected in the 14-day group providing evidence that vaccination can reduce transmission at $14 \mathrm{dpv}$ in this setting. In contrast, a study performed by Parida et al. (20), evaluated transmission and protection at 10 and $29 \mathrm{dpv}$. The oil-adjuvanted vaccine used was of high potency $\left(>18 \mathrm{PD}_{50}\right.$ based on cattle experiments) and contained the O Manisa strain. Challenge was through exposure by direct contact with pigs with clinical disease caused by the O UKG 34/2001 strain of serotype O. Of animals challenged at $10 \mathrm{dpv}, 13 / 16$ (81\%) were clinically diseased, while in the 29-day group, $2 / 8$ (25\%) were affected. In both groups, disease was reported to be milder and associated with reduced virus shedding compared to the unvaccinated control animals. Similar studies by Orsel et al. (31) aimed to assess transmission from infected, vaccinated pigs that had received O Manisa vaccine 14 days before challenge with O/NET/2001. They showed that vaccinated pigs could transmit infection to other vaccinated pigs as readily as to non-vaccinated controls. However, further work by the same group demonstrated that vaccination was able to reduce the transmission between pens (32). The differences reported in these studies could be attributed to different exposure methods, strains, or the small numbers of animals used.

Challenge studies were also performed to evaluate protection from an O Manisa vaccine to a strain from the O Mya98 lineage (33). Vaccines were double oil adjuvanted and $>6.0 \mathrm{PD}_{50}$ (presumably based on bovine challenge studies although this is not stated). Groups of five pigs were vaccinated and intradermally challenged at either 4 or $7 \mathrm{dpv}$. A non-vaccinated control group of five animals was also challenged for comparison. All control animals showed generalized disease. Four out of five (80\%) animals challenged at $7 \mathrm{dpv}$ were protected compared with three $(60 \%)$ animals challenged at $4 \mathrm{dpv}$ indicating animals may be protected soon after vaccination. Virus shedding was significantly lower in vaccinated animals compared to controls. Each group was in indirect contact (not physical but shared air handling unit) with five unvaccinated pigs to assess transmission in a controlled environment. No clinical signs or seroconversion was seen in pigs that were in contact with the vaccinated groups despite live virus being detected in the blood. This is in contrast to pigs that were in contact with the unvaccinated control animals although a breach in biosecurity may have explained this contrast. A similar study was performed by the same group using a serotype A Malaysia 97 vaccine and a serotype A/ASIA/Sea-97 lineage challenge strain (relationship value, $r_{1}$, around 0.5 ). Protection from generalized clinical disease was seen in all animals vaccinated 4 and 7 days pre challenge. No disease, FMD antibodies, or live virus was seen in the contact groups, although some animals in contact with the 4-day group were PCR positive on nasal swab (34).

In response to an FMD epidemic in Southeast Asia where there was only a moderate match between field and O Manisa vaccine strains ( $r_{1}$ around 0.3), Park et al. (35) performed homologous and heterologous challenge studies to evaluate a new vaccine seed strain (O/Andon/SKR/2010). Groups of five, FMD antibody-free, 3 -month-old pigs received one of three different antigen payloads $(7.5,10$, and $15 \mu \mathrm{g})$ in an oil-adjuvanted vaccine and were intradermally challenged $30 \mathrm{dpv}$ with the homologous O/Andon/ SKR/2010 strain. Two placebo injected pigs were challenged for comparison. All vaccinated animals were protected from clinical disease, ignoring any lesions seen at the inoculation site. Both control animals had generalized disease. The $10 \mu \mathrm{g}$ group was subsequently challenged with a heterologous strain of the ME-SA topotype ( $r_{1}$ value around 0.5$)$, and all animals were protected from clinical disease.

Challenge studies like those described can provide useful information on the potential role of vaccines in FMD control. There is evidence that protection may occur as early as 4 days, and vaccination may reduce transmission. Great care must be taken when extrapolating such results to a population level due to several factors including: variability in effective contact rates and virus shedding (quantity and duration) in the field; exposure routes and doses that have unclear relevance to field conditions; small sample sizes leading to uncertainty in the results from random error; and likely reduced responses to vaccination under program conditions. Therefore, these studies should be complimented by field-based epidemiological studies. Nowadays, decision making on how and when to use vaccination is greatly influenced by simulation studies with computer models. Unfortunately, it is not yet clear how to parameterize such models to make use of the results of potency tests.

\section{VACCINATION PROGRAMMES}

The level of immunity required to control disease at a population level depends in large part on the basic reproduction number $\left(\mathrm{R}_{0}\right)$ defined as the average number of secondary cases for each primary case in a completely susceptible population. The "effective" reproduction number is the same calculation but in a population with a proportion of immune individuals. If the effective reproduction number is less than one, on average, the circulation of infection will tend to reduce and ultimately cease. On this basis, the herd immunity required to bring the reproduction number to this level (called the "herd immunity threshold" or HIT) can be calculated by

$$
\mathrm{HIT}=1-\frac{1}{R_{0}}
$$


The basic reproduction number depends on the effective contact rate (i.e., contact between individuals sufficient for transmission per unit time, also known as the transmission parameter), duration of infectiousness, and population size (36). It is possible to estimate the duration of infectiousness from transmission studies although there is likely variation between viral strains and hosts (37). The effective contact rate is likely to be variable depending on environmental factors such as population or stocking density, production systems, season, and nature of any biosecurity practices. There is also the added complexity of population structures and the consideration of transmission at both the within and between herd level (38). $\mathrm{R}_{0}$ and the HIT can be estimated using mathematical models, although these should be parameterized as much as possible from field data and tailored to a specific country or region. Small-scale transmission studies can be used to parameterize models, but these should be validated from field-derived data to give greater confidence in model predictions.

The HIT is useful in giving a theoretical target for vaccination coverage (39). In pigs, maintaining sufficient population immunity through vaccination for FMD is a major challenge. Virus transmissibility is potentially high due to higher levels of virus excretion in this species (40), the intensive nature of modern pig production, and a rapid population turnover (particularly in fattening pigs typically slaughtered at 6-7 months old). Additionally, maternal antibodies interfere with the response to vaccines, and there is need for repeated doses of vaccine (discussed in detail in the following section). In some sub-populations with a high transmission risk, a relatively higher vaccination coverage is likely to be required making the case for risk-based vaccination targeting areas of high transmission identified using repeatable epidemiological methods.

\section{Vaccination Regimes}

Table 1 gives two proposed schedules for FMD vaccination in pigs both of which acknowledge the potential impact of maternally derived antibodies (MDA). Experiments have tried to address the issue of MDA interference with vaccination. Francis and Black (41) found that pigs as young as 1 week of age could mount a neutralizing antibody response to vaccine in the absence of MDA. They compared these responses to piglets with MDA from vaccinated sows and found that piglets aged between 1-4 weeks did not show any response with antibodies continuing to decline. An increase was seen in piglets vaccinated at 8 weeks old but was lower in the presence of higher levels of MDA. A recent study by Dekker et al. (42) assessed the serological response to vaccination in piglets at different ages (3-9 weeks) in the presence of MDA. Based on receiving a single dose and neutralizing titers 6 weeks post vaccination, the authors found that vaccination at 7-9 weeks old was optimal. Increases in titers were seen in all age categories although the responses were heavily dependent on the MDA level, which in turn was heavily influenced by the titer in the sow.

Two published studies from Taiwan have attempted to establish the optimal times and schedules for vaccination in pigs using field-derived serological evidence. Chung et al. (43) performed serological surveys as part of an active surveillance strategy on commercial pig farms with a herd size $\geq 5000$. Farms were using an oil-based, $>6.0 \mathrm{PD}_{50}$ serotype $\mathrm{O}$ vaccine. Two dose primary course schedules of 8 and 12 weeks, 10 and 14 weeks, and 12 and 16 weeks were compared through homologous neutralization tests on sera from 97 farms. This suggested that animals vaccinated at 12 and 16 weeks of age had the highest titers and there were significant differences between the vaccine products. This analysis was univariable and did not account for possible confounders and the time between vaccination and sampling. Liao et al. (44) performed a study whereby groups of between 6 and 15 piglets were vaccinated between 2 and 16 weeks old (some groups receiving a booster 4 weeks later). Based on neutralizing titers and homologous challenge studies, both performed at 24 weeks old, the authors suggested the optimum time for the first dose to be 8 weeks of age and titers were not significantly different if the piglet received a second dose at 12 weeks of age. This latter evidence for not needing a second dose based on antibody titers is contrary to the suggested schedules in Table $\mathbf{1}$.

\section{Routes of Administration}

Both of the vaccines listed in Table $\mathbf{1}$ are licensed for intramuscular administration in the neck region. Granulomas have been reported to occur in pigs at injection sites post vaccination with water-in-oil adjuvants (45). Although according to McKercher and Gailiunas (45) these were barely visible 6-12 months after vaccination, this could still be a problem in fattening pigs

TABLE 1 | Recommended schedules for commercially available oil-adjuvanted FMD vaccines licensed for use in pigs.

\begin{tabular}{|c|c|c|}
\hline $\begin{array}{l}\text { Product/ } \\
\text { Company }\end{array}$ & Schedules & Source \\
\hline AFTOPOR (Merial & Once at $2.5 \mathrm{mo}$ (if sporadic FMD cases in area) & "Guidance for Foot and Mouth \\
\hline Animal Health) & $\begin{array}{l}\text { Twice at } 2 \text { and } 3 \text { mo (Epizootics or highly virulent strain) } \\
>2 \text { wo if unvaccinated herd }\end{array}$ & $\begin{array}{l}\text { Disease Vaccination," Merial } \\
\text { Animal Health Limited }\end{array}$ \\
\hline $\begin{array}{l}\text { DECIVAC (MSD } \\
\text { Animal Health) }\end{array}$ & $\begin{array}{l}\text { Young animals with no maternal antibodies: primary dose }>2 \text { wo, second dose } 6 \text { weeks later in endemic areas. } \\
\text { Revaccination 4-6 months later } \\
\text { Young animals with maternal antibodies: primary dose 4-8 wo onward, second dose } 6 \text { weeks later in endemic } \\
\text { areas, with revaccination 4-6 months later } \\
\text { Adults: every } 6 \text { months }\end{array}$ & $\begin{array}{l}\text { http://www.msd-animal-health. } \\
\text { ph/products/131_118551/ } \\
\text { ProductDetails_131_118625. } \\
\text { aspx }\end{array}$ \\
\hline
\end{tabular}

Based on manufacturers listed at http://www.cfsph.iastate.edu/Vaccines/ (accessed August 9, 2016), where the company website states the schedule in English language and specifically for pigs. Both vaccines are licensed for intramuscular injection in the neck region.

wo, weeks old; mo, months old. 
slaughtered at 6-7 months of age where the neck region can have significant value. Such lesions have been reported to occur in $15-20 \%$ of pigs but could be easily removed by dissection at slaughter (46). Basarab et al. (47) reported 5/32 (16\%) pig carcases had large residual lesions after using a water-in-oil emulsion FMD vaccine requiring extensive dissection. These animals were vaccinated as weaners and the lesions were present at the end of the fattening period although the exact length of time between vaccination and slaughter is not reported. This same study found that intraperitoneal vaccination was equally efficacious to pigs vaccinated intramuscularly based on challenge studies but without the local tissue reaction. An experimental study by Eblé et al. (48) demonstrated that intradermal vaccination at $1 / 10$ th the dose of a normal killed vaccine was equally as effective based on challenge studies and neutralizing titers. The small numbers of animals in both of these studies may mean they were statistically underpowered, although both intraperitoneal and intradermal vaccination may offer significant advantages by reducing tissue lesions in fattening animals.

The issue of injection-site granulomas post vaccination has been particularly highlighted in the Republic of Korea and has been proposed as an important factor that has contributed to a reduced uptake of vaccination that has compromised coverage. As an example, a recent unpublished survey of 470 fattening crossbred pigs from four commercial farms found visually observable lesions in 87,80 , and $80 \%$ at 1,2 , and 3 months post vaccination, respectively. These were visible in live pigs, and all had received a two-dose primary course with the first dose given at 6-8 weeks of age and the second dose 2 weeks later. The injection site was in the neck approximately $2.5 \mathrm{~cm}$ caudal to the base of the ear. A subset of animals were slaughtered to demonstrate the gross pathology present as shown in Figure 2.

Although maintaining effective levels of coverage are challenging, a good understanding of the epidemiology will inform targeted vaccination strategies and more effective use of resources. The optimal vaccination schedules will vary depending

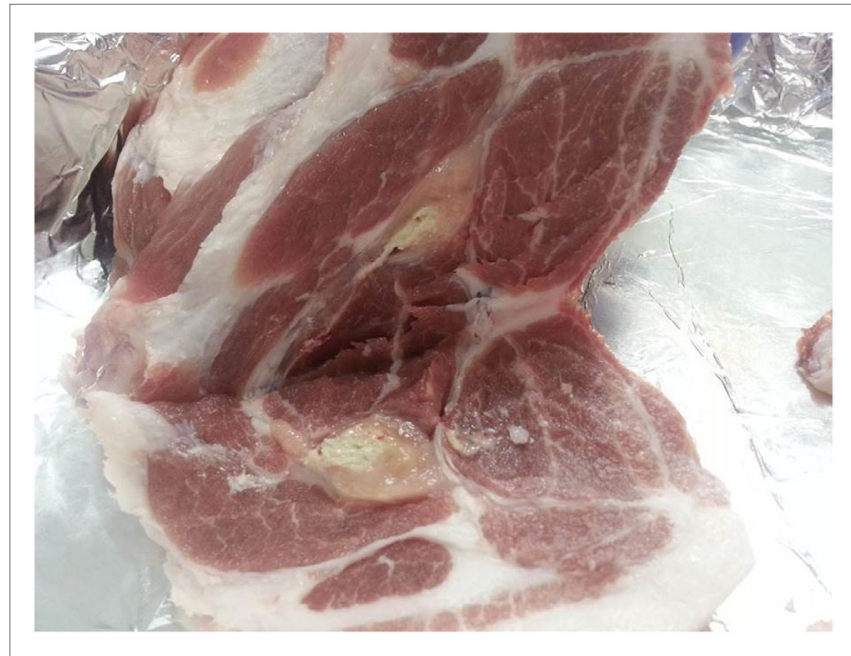

FIGURE 2 | Gross pathology lesion of an injection-site granuloma in the neck region of a pig from the Republic of Korea. on the antibody levels in the sow, which in turn will depend on vaccine type and schedules, natural exposure, and other sow- or piglet-related factors. Therefore, it is clear that countries embarking on vaccination programs should perform their own studies to establish optimal vaccination strategies as also suggested by Dekker et al. (42).

\section{NOVEL APPROACHES TO VACCINES AND VACCINATION}

Recent years have seen encouraging results with novel FMD vaccines and adjuvants. Those tested in pigs are considered briefly in this review and Table 2 summarizes some of the most promising challenge studies. Peptide vaccines for type O FMDV have been used in China for vaccination of pigs and continue to be improved. More data are needed on the breadth of crossprotection afforded by these vaccines against heterologous virus strains of the same serotype as used for peptide design. New vaccines have been designed, modified, and evaluated based upon FMD virus-like particles (VLP) generated in vitro or in the vaccinated pig through expression by virus vectors, especially adenoviruses. Specific methods of attenuating live FMDV now show considerable promise for overcoming the problem of combining inocuity with immunogenicity and can provide protection within 2 days. Data on duration of protection are awaited. IFNs and IFN inducers can not only provide extremely rapid and serotype non-specific protection against FMDV but they can also enhance the protection afforded by specific FMDV antigens and reduce the doses of adenovirus-vectored vaccines required for protection. New adjuvants have mostly been tested as additional incipients for oil-based vaccines and properly controlled and powered comparative studies of different adjuvants have not been published. There have been few recent studies of mucosal vaccine targeting or to evaluate DNA vaccines.

\section{Adenovirus-Vectored Vaccines}

Adenovirus-vectored FMD vaccines conditionally licensed in the USA in 2012 for use in cattle, have also shown efficacy in pigs. A replication-defective human serotype 5 adenovirus expressing the capsid encoding genes and the 3C protease needed for their cleavage and incorporating genetic material from the A24 strain of FMDV was given to pigs at a dose of $5 \times 10^{9} \mathrm{pfu}$, resulting in complete clinical protection against homologous FMDV by contact challenge at 7, 14, and $42 \mathrm{dpv}$ (56). It was later shown that a modified vector insert also expressing the FMDV $2 \mathrm{~B}$ gene improved the early antibody response to the FMDV capsid (57).

The same adenovirus vector when administered at high doses can deliver IFNs to provide early protection against FMDV and types I, II, and III IFN given this way can all provide protection to pigs for up to 5 days with evidence of synergistic action between different IFN types [reviewed by Stenfeldt et al. (58)]. Patch et al. (59) explored the possibility of selecting for a cytotoxic T cell response to FMDV in pigs vaccinated with an adenovirus expressing an inefficiently cleaved capsid precursor, but the protective value of this was not reported. 
TABLE 2 | Selected pig challenge study results with promising outcomes for novel vaccines.

\begin{tabular}{|c|c|c|c|c|}
\hline Vaccine & Vaccination $^{a}$ & Challenge & Protection & Reference \\
\hline \multirow[t]{2}{*}{$\begin{array}{l}\text { Live FMDV A12 attenuated by } \\
\text { Lpro mutation (A12-SAP) }\end{array}$} & $\begin{array}{l}15 \text { pigs vaccinated with } 10^{5}, 10^{6} \text {, or } \\
10^{7} \text { pfu } \mathrm{A} 12-\mathrm{SAP} \text { by subcutaneous } \\
\text { injection }\end{array}$ & $\begin{array}{l}\text { Intradermal heel bulb inoculation } \\
\text { with } 10^{5} \text { FMDV A12 at } 21 \mathrm{dpv}\end{array}$ & $\begin{array}{l}\text { All } 15 \text { pigs protected against clinical } \\
\text { signs (fever or vesicles), viremia, and } \\
\text { nasal shedding }\end{array}$ & (49) \\
\hline & $\begin{array}{l}9 \text { pigs vaccinated with } 10^{6} \text { pfu A12-SAP } \\
\text { by subcutaneous injection }\end{array}$ & $\begin{array}{l}\text { Intradermal heel bulb inoculation } \\
\text { with } 5 \times 10^{5} \text { FMDV A12 at 2, } 7 \text {, } \\
\text { or } 14 \mathrm{dpv}\end{array}$ & $\begin{array}{l}8 \text { of } 9 \text { pigs protected against clinical } \\
\text { signs }\end{array}$ & \\
\hline
\end{tabular}

Adenovirus vector expressing FMDV A24 P1-2A, 2B, 3B, 3C with Poly ICLC adjuvant in PBS

6 pigs vaccinated with $2.5 \times 10^{6}$ vector plus $1 \mathrm{mg}$ poly ICLC by subcutaneous injection of $2 \mathrm{ml}$ dose at 2 sites (other vaccination schedules evaluated)

Adenovirus vectors, one expressing porcine alpha and gamma interferons and the other expressing 3 small interfering RNAs

\section{5 minipigs vaccinated with $7.2 \times 10^{9}$ or} $1.7510^{10} \mathrm{TCID}_{50}$ of a combination of the adenovirus vectors (1:5 ratio of Ad-IFN titer to Ad-3siRNA titer) by intramuscular injection (other vaccination schedules evaluated)
Intradermal heel bulb inoculation with $10^{5}$ FMDV A24 at 7 or $21 \mathrm{dpv}$

All 3 pigs challenged at $21 \mathrm{dpv}$ protected against clinical signs, viremia, and nasal shedding (partial protection when challenged at $7 \mathrm{dpv}$ )

Direct contact of 5 groups of $3 \quad$ At the low "vaccine" dose, complete "vaccinated" minipigs at 2, 4, and clinical protection in 2/3, 1/3, and $7 \mathrm{dpv}$, for $18 \mathrm{~h}$ with donor minipigs $0 / 3$ minipigs at 2,4 , and $7 \mathrm{dpv}$. At infected with FMDV strain O/ the high "vaccine" dose it was 3/3 Andong/SKR/2010 and $1 / 3$ at 4 and $7 \mathrm{dpv}$. Viremia and oral shedding also reduced or prevented in some minipigs

45 pigs vaccinated in three groups of 15 pigs, each group consisting of 3 subgroups of 5 pigs receiving different doses: full $(2 \mathrm{ml}), 1 / 3$, or $1 / 9$ dose by volume intramuscularly. The full dose contained $300 \mu \mathrm{g}$ of epitope protein and $300 \mu$ p poly IC

5 pigs vaccinated with $10^{6} \mathrm{TCID}_{50}$ PRV-P12A3C in $2 \mathrm{ml}$ by intramuscular injection with identical booster at $21 \mathrm{dpv}$
Three potency tests involving challenge at $28 \mathrm{dpv}$ by intramuscular inoculation with $100050 \%$ infectious doses of one of three FMDV O strains: O/Mya/98, O/HN/CHA/93, O/

Tibet/99
$\mathrm{PD}_{50}$ results were $15.6(\mathrm{O} / \mathrm{Mya} / 98$ challenge), 15.6 (O/HN/CHA/93), and 7.0 (O/Tibet/99)
$100050 \%$ infectious doses of FMDV O/OR/80 by intramuscular inoculation at 15 days after booster vaccination
3 of 5 vaccinated pigs fully protected P1-2A, 3C from FMDV O ES/2001 (PRV-P12A3C) against clinical signs
$100050 \%$ infectious doses of FMDV Asia1/Jiangsu/China/2005 by intramuscular inoculation
All 5 vaccinated pigs fully protected against clinical signs
FMDV Asia1/Jiangsu/China/2005 5 pigs vaccinated with $50 \mu \mathrm{gg}$ VLP in oil VLP produced in E coli as SUMO-VPONP1NP3 fusion proteins, subsequently purified and cleaved
Dendrimeric B and T cell epitopes from FMDV O/ UKG/11/2001 adjuvant by intramuscular route 
disease virus fused to a FMDV T cell epitope from the $3 \mathrm{~A}$ viral non-structural protein. Intramuscular inoculation of pigs with this chimera and an oil adjuvant generated FMDV-specific cellmediated immunity and antibodies.

\section{Interferons}

Polyriboinosinic-polyribocytidylic acid stabilized with polyL-lysine and carboxymethyl cellulose (poly ICLC) is a synthetic double-stranded RNA (dsRNA) that is a viral mimic and activates multiple innate immune pathways through interaction with toll-like receptor 3 and MDA-5. It is a potent inducer of IFNs and can protect against FMD at 1 day after treatment (64). Its adjuvant affect on FMD vaccines in pigs was reported 40 years ago (65). Recently, it was shown to reduce, by 80 -fold, the dose required for protection of a recombinant adenovirus expressing FMDV A24 capsids [(50); Table 2]. Another synthetic analog of dsRNA, polyinosinic-polycytidylic acid (poly IC), potentiated the protection afforded by a multi-epitope vaccine in pigs (66). This vaccine incorporated linked $\mathrm{B}$ cell epitopes (the $\mathrm{G}-\mathrm{H}$ loop and $\mathrm{C}$ terminus of VP1) from four topotypes of serotype $\mathrm{O}$ flanked by two universal $\mathrm{T}$ cell epitopes. The final product in an oil adjuvant with poly IC protected pigs with $50 \%$ protection values of 7-16 against different challenge viruses [(52); Table 2].

\section{Other Adjuvants}

Barrette et al. (67) showed that intranasal immunization of pigs with detoxified Escherichia coli enterotoxins LTK63 and LTR72 linked to a peptide derived from the FMDV serotype O1-BFS VP1 G-H loop enhanced the antigen-specific mucosal and systemic immune responses to FMDV. Guo et al. (68) reported that a CpG-enriched plasmid enhanced the efficacy of a conventional FMD killed vaccine. Park et al. $(35,69)$ vaccinated groups of five pigs with a conventional FMD vaccine antigen plus either the oil adjuvant used in the Republic of Korea or with novel adjuvants (Carbigen, Emulsigen-D and ISA 201). In terms of immune response and post-challenge protection, the novel antigens were at least as good.

In a small field trial, administering $60 \mathrm{mg}$ of poly gamma glutamic acid (PGA) 3 days before FMDV vaccination of young pigs resulted in slightly more animals with detectable levels of FMDV antibodies 2-6 weeks later (70). Li et al. (71) reported increased antibody responses of pigs to a conventional FMDV vaccine supplemented with ginseng stem and leaf saponins. Xiao et al. (72) showed that an extract of the seeds of Momordica cochinchinensis (Lour.) Spreng. (ECMS) had a synergistic effect in improving the immune response of pigs after vaccination with inactivated FMDV antigens in an oil emulsion vaccine.

\section{Live Attenuated Vaccines}

Deleting the Lpro gene of FMDV A12 gave rise to an attenuated virus that partially protected pigs against wild-type challenge (73). Meanwhile, FMDV A24 lacking Lpro but with a capsid substituted from serotype $\mathrm{O}$ was still somewhat virulent for pigs. Changing the capsid genes to those of a cell culture adapted virus eliminated the virulence, but the resulting virus did not protect pigs when used as a vaccine (74). In contrast, mutating a conserved protein domain within the Lpro gene of FMDV A12 gave rise to a virus that was avirulent in pigs at a dose of $10^{7}$ but nevertheless elicited protection against FMDV challenge from $2 \mathrm{dpv}$ (49).

Codon bias deoptimization of the FMDV capsid-coding region (P1) introduced 489 nucleotide changes (19\%) but retained virus viability. The vaccine safety margin was $\sim 1000$-fold higher for pigs than for wild-type virus. Consistently, high levels of antibody titers were induced, even at the lowest dose tested (75).

\section{Protein/Peptide Vaccines}

Shao et al. (76) reported on the further development of a tandem repeat multiple-epitope recombinant vaccine against FMDV serotype $\mathrm{O}$ containing three copies of two VP1 epitopes of the O/China/99 strain of FMDV coupled with a porcine IgG heavy-chain constant region (77). This peptide vaccine elicited high titers of FMDV specific antibodies in pigs at $30 \mathrm{dpv}$ and conferred complete protection against a challenge with $100050 \%$ infective doses of the O/China/99 strain. Trials of another B cell epitope vaccine (52) have already been described above under IFNs (Table 2). Dong et al. (78) inserted the coding sequences of a FMDV serotype O VP1 epitope into a coliphage, resulting in an epitope-phage recombinant protein that formed a virus-like particle (VLP). Challenge inoculation of twice vaccinated pigs with the live homologous virus resulted in three of five animals being clinically protected from FMD.

Building upon earlier work $(79,80)$, Blanco et al. (55) reported that a synthetic dendrimeric peptide vaccine comprising two copies of a FMDV VP1 B cell epitope linked to a FMDV 3A T cell epitope protected pigs against disease and virus shedding after two doses of vaccination followed by challenge inoculation with live homologous FMDV O UK 2001 (Table 2). Guo et al. (54) have developed a bacterial expression system to generate VLPs of the FMDV Asia 1 capsid proteins. The FMDV genes VP0, VP1, and VP3 were each expressed as fusion products with the small ubiquitin like modifier protein (SUMO) and after removal of the SUMO moiety, the FMDV proteins assembled into VLPs. Five pigs vaccinated with $50 \mu \mathrm{g}$ of VLP emulsified in oil adjuvant were fully protected from challenge inoculation with live homologous FMDV (Table 2).

\section{DNA Vaccines}

DNA vaccines have not been completely effective in livestock despite promising results in mice. Multiple doses of plasmids expressing FMDV proteins or epitopes with coexpression of immunostimulants, and/or with conventional antigen boosters have been required to protect pigs against FMD (81-83). Most recently, Borrego et al. (84) reported partial protection of pigs after three immunizations with a DNA vaccine encoding FMDV $\mathrm{B}$ and $\mathrm{T}$ cell epitopes fused to the variable fragment of a mouse immunoglobulin against Class II swine leukocyte antigens.

\section{Mucosal Vaccines}

Although mucosal IgA may be elicited by parenteral immunization routes [e.g., Ref. (80)], mucosal vaccination might help to block FMDV entry. Barrette et al. (67) evaluated detoxified Escherichia coli enterotoxins LTK63 and LTR72 as mucosal adjuvants showing enhanced antigen-specific mucosal and systemic immunity for non-replicating antigens, including FMDV, upon intranasal 
immunization in pigs. Song et al. (85) reported vaccination of pigs with a recombinant VP1 epitope complex of serotype $\mathrm{O}$ FMDV fused to the cholera toxin B subunit (hCTB). Eight of ten pigs that were given three intraperitoneal immunizations were protected from challenge by inoculation with 106.5 TCID $_{50}$ type O FMDV. Wang et al. (86) showed that intranasal delivery of cationic PLGA nano/microparticles loaded with various FMDV DNA vaccine formulations encoding IL-6 as a molecular adjuvant enhanced protective immunity against FMDV, particularly pc-IL2AP12A3C with the IL-6 gene located before the P12A3C gene. Nevertheless, only partial protection against challenge with FMDV was achieved in pigs.

\section{Chimeric Killed Vaccines}

Blignaut et al. (87) produced a killed vaccine from a chimeric virus in which the capsid encoding genes were replaced with those from a different serotype. The resulting SAT 2 FMDV with a SAT 1 capsid were used to make a conventional killed vaccine that was potency tested in 17 pigs (three groups of five pigs given different vaccine doses and two unvaccinated control pigs). After a SAT 1 challenge by heel bulb inoculation, the $\mathrm{PD}_{50}$ was found to be $>6.4$. Zheng et al. (88) substituted the capsid-encoding region of a serotype $A$ virus vaccine for a more recent field isolate to update the antigenic match. The new vaccine was shown to protect against both the homologous strain and another semiheterologous one.

\section{RESEARCH PRIORITIES}

This review summarizes studies that have been undertaken to evaluate the performance of FMD vaccines in pigs, as well as introduce novel vaccination strategies that might be employed for FMD control in the future. Collectively, these data provide a valuable body of evidence that are especially relevant in the parts of the world where pigs play a central role in the maintenance and spread of the virus. Although a number of these experimental studies have evaluated the performance of FMDV vaccines, it is apparent that field data for such evaluation in pigs are currently lacking. Furthermore, much of this work is dependent upon bovine reagents, such as antigenic profiling (vaccine-matching), or exploits in vitro measurements of "correlates of protection" derived from cattle studies. In view of this paucity of data, when using vaccines in these settings, it is important to consider the different factors that influence whether, or not, a vaccine is likely to be efficacious. These include the (i) regime used (timing and frequency of vaccination); (ii) potency and formulation of oil vaccines; and (iii) antigenic match between the vaccine and circulating field strain. Although these three points are often assessed

\section{REFERENCES}

1. Knight-Jones TJD, Rushton J. The economic impacts of foot and mouth disease - what are they, how big are they and where do they occur? Prev Vet Med (2013) 112:161-73. doi:10.1016/j.prevetmed.2013.07.013

2. Leforban Y, Gerbier G. Review of the status of foot and mouth disease and approach to control/eradication in Europe and Central Asia. Rev Sci Tech (2002) 21(3):477-92. doi:10.20506/rst.21.3.1345 (and discussed) separately, they have an intimate relationship that underpins the performance of a vaccine. For example, it is usually accepted that a less than perfect antigenic match can be compensated by administration of a high potency vaccine; however, the impact of vaccine regime (as well as the herd-level coverage) is often ignored. In order to improve vaccine-induced immune responses, additional areas that warrant further scientific investigation include more systematic research to evaluate alternative vaccine adjuvants for vaccination in pigs, and research to validate of alternative routes (IM, IP, SC, ID) and sites of vaccination (to minimize local tissue granulomas in valuable meat cuts) and even multiple sites (with a divided dose). Effective (improved) vaccination regimes are also necessary to generate optimum protection in pigs to accommodate maternal antibody responses (to reduce the immunity gap).

Data from recent field outbreaks in Asia highlight the challenges posed by the control of FMD in pigs. While initiatives to improve the quality of vaccines and coverage that are tailored for pigs have the potential to make a positive impact on FMD control, it should be remembered that vaccination-alone is not a magic panacea and that FMD control, especially in the face of high amounts of circulating virus, is often reliant upon the implementation of effective zoo-sanitary (bio-containment) measures, as well as the maintenance of adequate local veterinary resources so that new clinical cases are rapidly investigated and detected.

\section{AUTHOR CONTRIBUTIONS}

NL, DK, and DP wrote extensive sections of the manuscript. NL led the structuring and format of the review. YL provided unique insights and data from the FMD situation in the Republic of Korea (the section of granulomas with image). All the authors read, edited, and approved the final manuscript.

\section{FUNDING}

The authors acknowledge the positive contributions made to the discussion forum at the Global Foot-and-Mouth Disease Research Alliance (GFRA) meeting held in Hanoi, Vietnam, in October 2015, and funding from UK Department for Environment, Food and Rural Affairs (Defra) and the European Commission for the control of foot-and-mouth disease (EuFMD). The Pirbright Institute receives grant-aided support from Biotechnology and Biological Sciences Research Council of the United Kingdom (BBS/E/I/00001713). The work of the WRLFMD is supported with funding provided to the EuFMD from the European Union. The views expressed herein can in no way be taken to reflect the official opinion of the European Union.

3. Clavijo A, Sanchez-Vazquez MJ, Buzanovsky LP, Martini M, Pompei JC, Cosivi O. Current status and future prospects to achieve foot-and-mouth disease eradication in South America. Transbound Emerg Dis (2015). doi:10.1111/ tbed.12345

4. Knowles NJ, He J, Shang Y, Wadsworth J, Valdazo-González B, Onosato $\mathrm{H}$, et al. Emergence of Southeast Asian foot-and-mouth disease viruses in East Asia. Emerg Infect Dis (2012) 18(3):499-501. doi:10.3201/ eid1803.110908 
5. Valdazo-González B, Timina A, Scherbakov A, Abdul-Hamid NF, Knowles NJ, King DP. Multiple introductions of serotype $\mathrm{O}$ foot-and-mouth disease viruses into East Asia in 2010-2011. Vet Res (2013) 44:76. doi:10.1186/12979716-44-76

6. Barteling SJ. Development and performance of inactivated vaccines against foot and mouth disease. Rev Sci Tech (2002) 21:577-88. doi:10.20506/ rst.21.3.1361

7. Doel TR. FMD vaccines. Virus Res (2003) 91:81-99. doi:10.1016/ S0168-1702(02)00261-7

8. Anderson EC, Masters RC, Mowat GN. Immune response of pigs to inactivated foot-and-mouth disease vaccines. Response to DEAE-dextran and saponin adjuvanted vaccines. Res Vet Sci (1971) 12:351-7.

9. Qiang Z, Huachun L. FMD vaccines and vaccination in China - production use and quality. International Conference on Scientific Developments and Technical Challenges in the Progressive Control of FMD in South Asia; 2011 Feb 13-15; New Delhi, India (2011).

10. Kitching P, Hammond J, Jeggo M, Charleston B, Paton D, Rodriguez L, et al. Global FMD control-Is it an option? Vaccine (2007) 25:5660-4. doi:10.1016/j. vaccine.2006.10.052

11. Paton DJ, Sumption KJ, Charleston B. Options for control of foot-and-mouth disease: knowledge, capability and policy. Philos Trans R Soc Lond B Biol Sci (2009) 364:2657-67. doi:10.1098/rstb.2009.0100

12. Sumption K. The global control of FMD. Open Session of the EuFMD Standing Technical Committee; 2008 Oct 14-17. Erice, Sicily (2008).

13. Heininger U, Bachtiar NS, Bahri P, Dana A, Dodoo A, Gidudu J, et al. The concept of vaccination failure. Vaccine (2012) 30:1265-8. doi:10.1016/j. vaccine.2011.12.048

14. Chen SP, Sun YF, Lee MC, Cheng IC, Yang PC, Huang TS, et al. Immune responses to foot-and-mouth disease virus in pig farms after the 1997 outbreak in Taiwan. Vet Microbiol (2008) 126:82-90. doi:10.1016/j.vetmic.2007.07.007

15. Black L, Francis MJ, Rweyemamu MM, Umebara O, Boge A. The relationship between serum antibody titres and protection from foot and mouth disease in pigs after oil emulsion vaccination. J Biol Stand (1984) 12:379-89. doi:10.1016/ S0092-1157(84)80062-1

16. Cox SJ, Gubbins S, Barnett PV. IL-6 production following vaccination in pigs - an additional immune response parameter for assessing FMD vaccine efficacy? Vaccine (2011) 29:4704-8. doi:10.1016/j.vaccine.2011.04.100

17. Zhang L, Feng X, Jin Y, Ma J, Cai H, Zhang X. Immunoprotective mechanisms in swine within the "grey zone" in antibody response after immunization with foot-and-mouth disease vaccine. Virus Res (2016) 220:39-46. doi:10.1016/ j.virusres.2016.04.008

18. Eblé PL, Bouma A, Weerdmeester K, Stegeman JA, Dekker A. Serological and mucosal immune responses after vaccination and infection with FMDV in pigs. Vaccine (2007) 25:1043-54. doi:10.1016/j.vaccine.2006.09.066

19. Cox SJ, Aggarwal N, Statham RJ, Barnett PV. Longevity of antibody and cytokine responses following vaccination with high potency emergency FMD vaccines. Vaccine (2003) 21:1336-47. doi:10.1016/S0264-410X(02)00691-6

20. Parida S, Fleming L, Oh Y, Mahapatra M, Hamblin P, Gloster J, et al. Reduction of foot-and-mouth disease (FMD) virus load in nasal excretions, saliva and exhaled air of vaccinated pigs following direct contact challenge. Vaccine (2007) 25:7806-17. doi:10.1016/j.vaccine.2007.08.058

21. Salt JS, Barnett PV, Dani P, Williams L. Emergency vaccination of pigs against foot-and-mouth disease: protection against disease and reduction in contact transmission. Vaccine (1998) 16:746-54. doi:10.1016/S0264-410X(97)86180-4

22. Barnard AL, Arriens A, Cox S, Barnett P, Kristensen B, Summerfield A, et al. Immune response characteristics following emergency vaccination of pigs against foot-and-mouth disease. Vaccine (2005) 23:1037-47. doi:10.1016/j. vaccine.2004.07.034

23. Barnett PV, Cox SJ, Aggarwal N, Gerber H, McCullough KC. Further studies on the early protective responses of pigs following immunisation with high potency foot and mouth disease vaccine. Vaccine (2002) 20:3197-208. doi:10.1016/S0264-410X(02)00242-6

24. Rigden RC, Carrasco CP, Barnett PV, Summerfield A, McCullough KC. Innate immune responses following emergency vaccination against footand-mouth disease virus in pigs. Vaccine (2003) 21:1466-77. doi:10.1016/ S0264-410X(02)00663-1

25. Eblé PL, de Bruin MGM, Bouma A, van Hemert-Kluitenberg F, Dekker A. Comparison of immune responses after intra-typic heterologous and homologous vaccination against foot-and-mouth disease virus infection in pigs. Vaccine (2006) 24:1274-81. doi:10.1016/j.vaccine.2005.09.040

26. Garcia-Briones MM, Blanco E, Chiva C, Andreu D, Ley V, Sobrino F. Immunogenicity and $\mathrm{T}$ cell recognition in swine of foot-and-mouth disease virus polymerase 3D. Virology (2004) 322:264-75. doi:10.1016/j. virol.2004.01.027

27. World Organisations for Animal Health (OIE). Foot and mouth disease. Manual of Diagnostic Tests and Vaccines for Terrestrial Animals (Version Adopted in May 2012). Paris: OIE (2015). p. 145-73.

28. European Directorate for the Quality of Medicines. Foot-and-mouth disease (ruminants) vaccine (inactivated). European Pharmacopoeia. Version 5.5. 04/2005:0063. Strasbourg, France: Council of Europe (2006).

29. Li D, Lu Z-J, Xie B-X, Sun P, Chen Y-L, Fu Y-F, et al. Alternative way to test the efficacy of swine FMD vaccines: measurement of pigs median infected dose (PID50) and regulation of live virus challenge dose. Virol J (2010) 7:215. doi:10.1186/1743-422X-7-215

30. Eblé PL, Bouma A, De Bruin MGM, Van Hemert-Kluitenberg F, Van Oirschot JT, Dekker A. Vaccination of pigs two weeks before infection significantly reduces transmission of foot-and-mouth disease virus. Vaccine (2004) 22:1372-8. doi:10.1016/j.vaccine.2003.11.003

31. Orsel K, de Jong MCM, Bouma A, Stegeman JA, Dekker A. Foot and mouth disease virus transmission among vaccinated pigs after exposure to virus shedding pigs. Vaccine (2007) 25:6381-91. doi:10.1016/j.vaccine.2007. 06.010

32. van Roermund HJW, Eble PL, de Jong MCM, Dekker A. No between-pen transmission of foot-and-mouth disease virus in vaccinated pigs. Vaccine (2010) 28:4452-61. doi:10.1016/j.vaccine.2010.04.019

33. Vosloo W, Hong NTT, Geoffrey FT, Jacqueline MM, Jianning W, Van Phuc K, et al. Efficacy of a high potency O1 Manisa monovalent vaccine against heterologous challenge with a FMDV O Mya98 lineage virus in pigs 4 and 7 days post vaccination. Vaccine (2015) 33:2778-85. doi:10.1016/j.vaccine.2015.04.045

34. Nagendrakumar SB, Hong NTT, Geoffrey FT, Jacqueline MM, Andrew D, Michelle G, et al. A Malaysia 97 monovalent foot-and-mouth disease vaccine (\&gt;6PD50/dose) protects pigs against challenge with a variant FMDV A SEA-97 lineage virus, 4 and 7 days post vaccination. Vaccine (2015) 33:1-7. doi:10.1016/j.vaccine.2015.07.014

35. Park JN, Lee SY, Chu JQ, Lee YJ, Kim RH, Lee KN, et al. Protection to homologous and heterologous challenge in pigs immunized with vaccine against footand-mouth disease type O caused an epidemic in East Asia during 2010/2011. Vaccine (2014) 32:1882-9. doi:10.1016/j.vaccine.2014.01.067

36. Vynnycky E, White RG. How are models set up? An Introduction to Infectious Disease Modelling. New York: Oxford University Press (2010). p. 13-40.

37. Kinsley AC, Patterson G, VanderWaal KL, Craft ME, Perez AM. Parameter values for epidemiological models of foot-and-mouth disease in swine. Front Vet Sci (2016) 3:44. doi:10.3389/fvets.2016.00044

38. Hagenaars TJ, Dekker A, de Jong MCM, Eblé PL. Estimation of foot and mouth disease transmission parameters, using outbreak data and transmission experiments. Rev Sci Tech (2011) 30:467-77.

39. Fine PEM. The basics: infections, transmission and models. In: Vynnycky E, White R, editors. An Introduction to Infectious Disease Modelling. New York: Oxford University Press (2010). p. 1-12.

40. Alexandersen S, Quan M, Murphy C, Knight J, Zhang Z. Studies of quantitative parameters of virus excretion and transmission in pigs and cattle experimentally infected with foot-and-mouth disease virus. J Comp Pathol (2003) 129:268-82. doi:10.1016/S0021-9975(03)00045-8

41. Francis MJ, Black L. Response of young pigs to foot-and-mouth disease oil emulsion vaccination in the presence and absence of maternally derived neutralising antibodies. Res Vet Sci (1986) 41:33-9.

42. Dekker A, Chénard G, Stockhofe N, Eblé PL. Proper timing of foot-and-mouth disease vaccination of piglets with maternally derived antibodies will maximize expected protection levels. Front Vet Sci (2016) 3:10-5. doi:10.3389/ fvets.2016.00052

43. Chung WB, Liao PC, Chen SP, Yang PC, Lin YL, Jong MH, et al. Optimization of foot-and-mouth disease vaccination protocols by surveillance of neutralization antibodies. Vaccine (2002) 20:2665-70. doi:10.1016/S0264-410X(02)00201-3

44. Liao PC, Lin YL, Jong MH, Chung WB. Efficacy of foot-and-mouth disease vaccine in pigs with single dose immunization. Vaccine (2003) 21:1807-10. doi:10.1016/S0264-410X(03)00030-6 
45. McKercher PD, Gailiunas P. Response of swine to inactivated foot-and-mouth disease vaccine. Duration of immunity and local tissue reaction. Arch für die gesamte Virusforsch (1969) 28:165-76. doi:10.1007/BF01249381

46. Nöckler A, Thalmann G, Haack P, Nehmzow P, Poewe R, Pietzsch E, et al. Riems foot-and-mouth disease oil emulsion vaccines for swine. 2. Use of foot-and-mouth 2-component oil emulsion vaccines in practice. Arch für Exp Veterinärmedizin (1990) 44:183-8.

47. Basarab $\mathrm{O}$, Umehara $\mathrm{O}$, Uribe $\mathrm{L}$. The protection of fattening pigs against foot and mouth disease with an oil-adjuvant vaccine: studies on South American FMD virus strains. Rev Sci Tech (1982) 1:1147-54.

48. Eblé PL, Weerdmeester K, van Hemert-Kluitenberg F, Dekker A. Intradermal vaccination of pigs against FMD with 1/10 dose results in comparable vaccine efficacy as intramuscular vaccination with a full dose. Vaccine (2009) 27:1272-8. doi:10.1016/j.vaccine.2008.12.011

49. Díaz-San Segundo F, Weiss M, Pérez-Martín E, Dias CC, Grubman MJ, Santos TDL. Inoculation of swine with foot-and-mouth disease SAP-mutant virus induces early protection against disease. J Virol (2012) 86:1316-27. doi:10.1128/JVI.05941-11

50. Díaz-San Segundo F, Dias CC, Moraes MP, Weiss M, Perez-Martin E, Salazar AM, et al. Poly ICLC increases the potency of a replication-defective human adenovirus vectored foot-and-mouth disease vaccine. Virology (2014) 468:283-92. doi:10.1016/j.virol.2014.08.012

51. Kim S-M, Park J-H, Lee K-N, Kim S-K, You S-H, Kim T, et al. Robust protection against highly virulent foot-and-mouth disease virus in swine by combination treatment with recombinant adenoviruses expressing porcine alpha and gamma interferons and multiple small interfering RNAs. J Virol (2015) 89:8267-79. doi:10.1128/JVI.00766-15

52. Cao Y, Lu Z, Li D, Fan P, Sun P, Bao H, et al. Evaluation of cross-protection against three topotypes of serotype $\mathrm{O}$ foot-and-mouth disease virus in pigs vaccinated with multi-epitope protein vaccine incorporated with poly(I: C). Vet Microbiol (2014) 168:294-301. doi:10.1016/j.vetmic.2013.11.023

53. Zhang $\mathrm{K}$, Huang J, Wang Q, He Y, Xu Z, Xiang M, et al. Recombinant pseudorabies virus expressing P12A and $3 \mathrm{C}$ of FMDV can partially protect piglets against FMDV challenge. Res Vet Sci (2011) 91:90-4. doi:10.1016/ j.rvsc.2010.09.001

54. Guo HC, Sun SQ, Jin Y, Yang SL, Wei YQ, Sun DH, et al. Foot-and-mouth disease virus-like particles produced by a SUMO fusion protein system in Escherichia coli induce potent protective immune responses in guinea pigs, swine and cattle. Vet Res (2013) 44:1. doi:10.1186/1297-9716-44-48

55. Blanco E, Guerra B, de la Torre BG, Defaus S, Dekker A, Andreu D, et al. Full protection of swine against foot-and-mouth disease by a bivalent B-cell epitope dendrimer peptide. Antiviral Res (2016) 129:74-80. doi:10.1016/ j.antiviral.2016.03.005

56. Moraes MP, Mayr GA, Mason PW, Grubman MJ. Early protection against homologous challenge after a single dose of replication-defective human adenovirus type 5 expressing capsid proteins of foot-and-mouth disease virus (FMDV) strain A24. Vaccine (2002) 20:1631-9. doi:10.1016/ S0264-410X(01)00483-2

57. Pena L, Moraes MP, Koster M, Burrage T, Pacheco JM, Segundo FDS, et al. Delivery of a foot-and-mouth disease virus empty capsid subunit antigen with nonstructural protein 2B improves protection of swine. Vaccine (2008) 26:5689-99. doi:10.1016/j.vaccine.2008.08.022

58. Stenfeldt C, Diaz-San Segundo F, de Los Santos T, Rodriguez LL, Arzt J. The pathogenesis of foot-and-mouth disease in pigs. Front Vet Sci (2016) 3:41. doi:10.3389/fvets.2016.00041

59. Patch JR, Pedersen LE, Toka FN, Moraes M, Grubman MJ, Nielsen M, et al. Induction of foot-and-mouth disease virus-specific cytotoxic $\mathrm{T}$ cell killing by vaccination. Clin Vaccine Immunol (2011) 18:280-8. doi:10.1128/ CVI.00417-10

60. Liu Y, Hu R, Zhang S, Zhang F, Li Z, Wei X, et al. Expression of the Foot-and-Mouth Disease Virus VP1 protein using a replication-competent recombinant canine adenovirus type 2 elicits a humoral antibody response in a porcine model. Viral Immunol (2006) 19:202-9. doi:10.1089/vim.2006.19.202

61. Qian P, Li XM, Jin ML, Peng GQ, Chen HC. An approach to a FMD vaccine based on genetic engineered attenuated pseudorabies virus: one experiment using VP1 gene alone generates an antibody responds on FMD and pseudorabies in swine. Vaccine (2004) 22:2129-36. doi:10.1016/j.vaccine.2003.12.005
62. Yang C-D, Liao J-T, Lai C-Y, Jong M-H, Liang C-M, Lin Y-L, et al. Induction of protective immunity in swine by recombinant bamboo mosaic virus expressing foot-and-mouth disease virus epitopes. BMC Biotechnol (2007) 7:62. doi:10.1186/1472-6750-7-62

63. Crisci E, Fraile L, Moreno N, Blanco E, Cabezón R, Costa C, et al. Chimeric calicivirus-like particles elicit specific immune responses in pigs. Vaccine (2012) 30:2427-39. doi:10.1016/j.vaccine.2012.01.069

64. Dias CC, Moraes MP, Weiss M, Diaz-San Segundo F, Pérez-Martín E, Salazar AM, et al. Novel antiviral therapeutics to control foot-and-mouth disease. J Interferon Cytokine Res (2012) 32:462-73. doi:10.1089/jir.2012. 0012

65. Cunliffe HR, Richmond JY, Campbell CH. Interferon inducers and footand-mouth disease vaccines: influence of two synthetic polynucleotides on antibody response and immunity in guinea pigs and swine. Can J Comp Med (1977) 41:117-21.

66. Cao Y, Lu Z, Li Y, Sun P, Li D, Li P, et al. Poly(I: C) combined with multi-epitope protein vaccine completely protects against virulent foot-and-mouth disease virus challenge in pigs. Antiviral Res (2013) 97:145-53. doi:10.1016/j. antiviral.2012.11.009

67. Barrette RW, Szczepanek SM, Rood D, Challa S, Avery N, Vajdy M, et al. Use of inactivated Escherichia coli enterotoxins to enhance respiratory mucosal adjuvanticity during vaccination in swine. Clin Vaccine Immunol (2011) 18:1996-8. doi:10.1128/CVI.05273-11

68. Guo X, Jia H, Zhang Q, Yuan W, Zhu G, Xin T, et al. CpG-enriched plasmid enhances the efficacy of the traditional foot-and-mouth disease killed vaccine. Microbiol Immunol (2012) 56:332-7. doi:10.1111/j.1348-0421.2012.00438.x

69. Park ME, Lee SY, Kim RH, Ko MK, Lee KN, Kim SM, et al. Enhanced immune responses of foot-and-mouth disease vaccine using new oil/gel adjuvant mixtures in pigs and goats. Vaccine (2014) 32:5221-7. doi:10.1016/j. vaccine.2014.07.040

70. Lee J-H, Kang I-J, Kim A-R, Noh Y-S, Chung H-C, Park B-K. Increased humoral antibody response of foot-and-mouth disease virus vaccine in growing pigs pre-treated with poly- $\gamma$-glutamic acid. J Vet Sci (2016) 17:253-6. doi:10.4142/jvs.2016.17.2.253

71. Li Y, Xie F, Chen J, Fan Q, Zhai L, Hu S. Increased humoral immune responses of pigs to foot-and-mouth disease vaccine supplemented with ginseng stem and leaf saponins. Chem Biodivers (2012) 9:2225-35. doi:10.1002/cbdv. 201100377

72. Xiao C, Rajput ZI, Liu D, Hu S. Enhancement of serological immune responses to foot-and-mouth disease vaccine by a supplement made of extract of cochinchina momordica seeds. Clin Vaccine Immunol (2007) 14:1634-9. doi:10.1128/CVI.00339-07

73. Chinsangaram J, Mason PW, Grubman MJ. Protection of swine by live and inactivated vaccines prepared from a leader proteinase-deficient serotype A12 foot-and-mouth disease virus. Vaccine (1998) 16:1516-22. doi:10.1016/ S0264-410X(98)00029-2

74. Almeida MR, Rieder E, Chinsangaram J, Ward G, Beard C, Grubman MJ, et al. Construction and evaluation of an attenuated vaccine for foot-andmouth disease: difficulty adapting the leader proteinase-deleted strategy to the serotype O1 virus. Virus Res (1998) 55:49-60. doi:10.1016/S0168-1702(98) 00031-8

75. Diaz-San Segundo F, Medina GN, Ramirez-Medina E, Velazquez-Salinas L, Koster M, Grubman MJ, et al. Synonymous deoptimization of foot-andmouth disease virus causes attenuation in vivo while inducing a strong neutralizing antibody response. J Virol (2016) 90:1298-310. doi:10.1128/ JVI.02167-15

76. Shao JJ, Wong CK, Lin T, Lee SK, Cong GZ, Sin FWY, et al. Promising multiple-epitope recombinant vaccine against foot-and-mouth disease virus type o in swine. Clin Vaccine Immunol (2011) 18:143-9. doi:10.1128/CVI. 00236-10

77. Chan EW, Wong HT, Cheng SC, Yan WY, Zheng ZX, Sheng ZT, et al. An immunoglobulin $\mathrm{G}$ based chimeric protein induced foot-and-mouth disease specific immune response in swine. Vaccine (2000) 19:538-46. doi:10.1016/ S0264-410X(00)00186-9

78. Dong YM, Zhang GG, Huang XJ, Chen L, Chen HT. Promising MS2 mediated virus-like particle vaccine against foot-and-mouth disease. Antiviral Res (2015) 117:39-43. doi:10.1016/j.antiviral.2015.01.005 
79. Cubillos C, de la Torre BG, Bárcena J, Andreu D, Sobrino F, Blanco E. Inclusion of a specific $\mathrm{T}$ cell epitope increases the protection conferred against foot-and-mouth disease virus in pigs by a linear peptide containing an immunodominant B cell site. Virol J (2012) 9:66. doi:10.1186/1743422X-9-66

80. Cubillos C, de la Torre BG, Jakab A, Clementi G, Borrás E, Bárcena J, et al. Enhanced mucosal immunoglobulin A response and solid protection against foot-and-mouth disease virus challenge induced by a novel dendrimeric peptide. J Virol (2008) 82:7223-30. doi:10.1128/JVI.00401-08

81. Huang H, Yang Z, Xu Q, Sheng Z, Xie Y, Yan W, et al. Recombinant fusion protein and DNA vaccines against foot and mouth disease virus infection in guinea pig and swine. Viral Immunol (1999) 12:1-8. doi:10.1089/ vim.1999.12.1

82. Li Y, Stirling CMA, Denyer MS, Hamblin P, Hutchings G, Takamatsu HH, et al. Dramatic improvement in FMD DNA vaccine efficacy and cross-serotype antibody induction in pigs following a protein boost. Vaccine (2008) 26:2647-56. doi:10.1016/j.vaccine.2008.01.037

83. Wong HT, Cheng SCS, Chan EWC, Sheng ZT, Yan WY, Zheng ZX, et al. Plasmids encoding foot-and-mouth disease virus VP1 epitopes elicited immune responses in mice and swine and protected swine against viral infection. Virology (2000) 278:27-35. doi:10.1006/viro.2000.0607

84. Borrego B, Argilaguet JM, Pérez-Martín E, Dominguez J, Perez-Filgueira M, Escribano JM, et al. A DNA vaccine encoding foot-and-mouth disease virus $\mathrm{B}$ and T-cell epitopes targeted to class II swine leukocyte antigens protects pigs against viral challenge. Antiviral Res (2011) 92:359-63. doi:10.1016/ j.antiviral.2011.07.017
85. Song H, Wang Z, Zheng D, Fang W, Li Y, Liu Y, et al. A novel mucosal vaccine against foot-and-mouth disease virus induces protection in mice and swine. Biotechnol Lett (2005) 27:1669-74. doi:10.1007/s10529-005-2727-4

86. Wang G, Pan L, Zhang Y, Wang Y, Zhang Z, Lü J, et al. Intranasal delivery of cationic PLGA nano/microparticles-loaded fmdv DNA vaccine encoding IL-6 elicited protective immunity against FMDV challenge. PLoS One (2011) 6:e27605. doi:10.1371/journal.pone.0027605

87. Blignaut B, Visser N, Theron J, Rieder E, Maree FF. Custom-engineered chimeric foot-and-mouth disease vaccine elicits protective immune responses in pigs. J Gen Virol (2011) 92:849-59. doi:10.1099/vir.0.027151-0

88. Zheng H, Lian K, Yang F, Jin Y, Zhu Z, Guo J, et al. Cross-protective efficacy of engineering serotype $A$ foot-and-mouth disease virus vaccine against the two pandemic strains in swine. Vaccine (2015) 33:5772-8. doi:10.1016/j. vaccine.2015.09.055

Conflict of Interest Statement: The authors declare that the research was conducted in the absence of any commercial or financial relationships that could be construed as a potential conflict of interest.

Copyright (c) 2016 Lyons, Lyoo, King and Paton. This is an open-access article distributed under the terms of the Creative Commons Attribution License (CC BY). The use, distribution or reproduction in other forums is permitted, provided the original author(s) or licensor are credited and that the original publication in this journal is cited, in accordance with accepted academic practice. No use, distribution or reproduction is permitted which does not comply with these terms. 